\title{
Does a Circadian Variation Occur in Myocardial Ischemia over 48 hours in Patients with Unstable Angina?
}

\author{
Luiz A ntônio Machado César, João Fernando Monteiro Ferreira, César J. Grupi, David Pamplona, \\ Miguel A. Moretti, Elcio Pferfmann, José A. F. Ramires
}

São Paulo, SP - Brazil

\begin{abstract}
Objective - To study the incidence of and variation in myocardial ischemia over 48 hours in patients with unstable angina.
\end{abstract}

Methods - Thirty-nine patients with unstable angina underwent long-term electrocardiography for 48 hours. The number of events and the period of time of ischemia (in minutes) were analyzed for the 48 hours, in two periods of 24 hours, and in periods of 4 hours.

Results - We analyzed 1755.8 hours of monitoring tapes, and ischemic episodes were detected in 18 (46.2\%) patients, corresponding to 173 ischemic episodes, allowing the evaluation of 1304 minutes of ischemia.only 4 of which were $(2.2 \%)$ symptomatic, Considering the entire period of time of recording and the predetermined time intervals, we observed a higher number of ischemic episodes (38) and a longer duration of ischemia (315.4 minutes) between 11:00 am and 3:00 pm. However, no significant differences occurred among the values in the different intervals.

Conclusion - Long-term electrocardiography over 48 hours showed a high incidence (97.8\%) of silent ischemic episodes in patients with unstable angina. No evidence of a circadian variation of myocardial ischemia in unstable angina was observed.

Key words: myocardial ischemia, Holter monitoring, unstable angina.
Heart Institute, Hospital das Clínicas - FMUSP

Mailing address: Luiz Antônio Machado César - InCor - Unidade de Coronariopatias Crônicas - Av. Dr. Enéas C. Aguiar, 44 - 05403-000 - São Paulo, SP
Myocardial ischemia is the result of inadequate coronary blood flow, promoting alterations in cellular metabolism, myocardial relaxation, and contractility, followed by ST-Tsegment abnormalities on electrocardiogram (ST- T segment elevation or depression), culminating in clinical manifestation, i.e., angina.

Ischemic events can occur without clinical manifestations; in fact, most of them (60-90\%) are silent ${ }^{1-4}$. Based on this fact, the concept of silent ischemia arose, i.e., the occurrence of ischemic events without any clinical expression that could identify them.

Long-term electrocardiography, due, is the most used method in clinical studies for evaluating the presence of myocardial ischemia, particularly in individuals with documented coronary artery disease ${ }^{5}$. Using this method, a number of studies have been conducted involving individuals with known coronary artery disease and clinical manifestations of angina. In 1982, Johnson et $\mathrm{al}^{6}$, studying patients with unstable angina, reported the importance of the presence of silent ischemia identifying those with more extensive coronary artery disease and those subgroups with a worse short-term clinical course. Similarly, Gotlieb et $\mathrm{al}^{7}$ showed the importance of myocardial ischemia as a prognostic factor during the first 30 days in patients with unstable angina and, even in those patients who received intensive pharmacological treatment, the presence of ischemia had a prognostic value higher than coronary anatomy evaluated by angiography.

Another interesting phenomenon is the variation in ischemic events throughout the 24 hours of a day. Reports exist indicating that ischemia, with or without symptoms, has a circadian variation in either stable ${ }^{8,9}$ or unstable angina ${ }^{10-13}$. Moreover, we know neither the incidence of silent ischemia in individuals with unstable angina nor its variation throughout the 24 hours of a day in our patient population.

Therefore, the purpose of this study was to investigate the features of myocardial ischemia, evaluated with longterm electrocardiography, with regard to the incidence and variation of ischemia over 48 hours in patients with unstable angina. 


\section{Methods}

We studied 39 patients, 21 males and 18 females, with a mean age of $58 \pm 10.71$ years (38-75 years) and a diagnosis of unstable angina, who were treated at the Emergency Unit of the Heart Institute of the Hospital das Clínicas of FMUSP, from November 1993 to December 1995. At the first screening, the clinical history, a physical examination, a standard 12-lead surface electrocardiogram, plasma levels of creatine kinase (CK-MB), BUN, creatinine, glucose, and blood cell count were obtained. All the patients underwent long-term electrocardiography (Holter monitoring) for 48 consecutive hours. From the beginning and during the entire study period, the patients received aspirin $100 \mathrm{mg} /$ day orally, diazepam $5 \mathrm{mg}$ each 8 hours orally and intravenous nitrate.

We required two criteria for the diagnosis of unstable angina: 1) clinical criterion - unstable angina as a manifestation of chest discomfort or pain, radiating or not to one or both upper limbs, neck, dorsal region, epigastrium or jaw, accompanied or not by concomitant factors, such as sweating, palpitations or sense of imminent death. It was necessary for the diagnosis that the patient had experienced a clear worsening of symptoms for no more than 7 days and the last episode for no more than 24 hours; 2) electrocardiographic criterion - it was necessary that the electrocardiogram had at least one of the following alterations: transient or persistent $\mathrm{T}$-wave inversion in at least two contiguous leads, or transient ST-segment depression $>1 \mathrm{~mm}$ in two or more contiguous leads.

The exclusion criteria were postinfarction angina, previous cardiac surgery or coronary angioplasty, valvular heart disease, uncontrolled arterial hypertension, congestive heart failure, hepatic and renal failure, uncontrolled diabetes mellitus, signs and symptoms of hyperthyroidism, anemia, pregnancy and age $<18$ years or $>80$ years, as well as alterations on the 12 lead electrocardiogram, such as right or left bundle branch block, Mobitz type II $2^{\text {nd }}$ degree atrioventricular block, $3^{\text {rd }}$ degree atrioventricular block, PR interval $<0.10$ second, delta wave indicative of presence an accessory pathway, even in the absence of Wolf-ParkinsonWhite syndrome, atrial fibrillation, atrial flutter, and narrow or wide QRS tachycardias.

Long-term electrocardiography - technical considerations about the examination and recordings - a two-channel $\left(\mathrm{CM}_{5}\right.$ and $\left.\mathrm{CM}_{1} \mathrm{M}\right)$ portable Marquette 8000 monitor was used (Marquette, Milwaukee, WI, USA), with wave recording in AM (amplitude-modulated), containing a cassette tape for continuous 24-hour recording. After this time, the tape was replaced by another allowing a total of 48 hours of study. The monitor was connected to the patients and during the following 48 hours the patients were instructed to report the occurrence of chest pain or episodes of discomfort similar to those that had caused them to go to the hospital. The time of any pain episode was registered in the diary of the long-term electrocardiography examination, in order to verify whether a certain ischemic event had been symptomatic or not. After the monitor was removed, the tapes were analyzed on the processor Marquette Laser SXP processor (Marquette, Milwaukee, WI, USA, 1988) and assessed by at least two professionals of the Electrocardiology Unit of the Heart Institute.

Diagnostic criteria for ischemic events: ST-segment elevation $>2 \mathrm{~mm}$ or horizontal or downsloping ST-segment depression $>1 \mathrm{~mm}$, persisting for at least 1 minute, and returning to the baseline for at least 1 minute. The possible events identified as described were defined as ischemic episodes, and each one of these events was registered on paper, with a velocity of $25 \mathrm{~mm} / \mathrm{second}$, with the pattern of 1 $\mathrm{mV}$, using a Marquette laser printer, and then ST-segment variation and the period of time of ischemia of each event were reevaluated manually.

Evaluation of ischemic events: 1) the total number of episodes and the sum of the period of time in minutes, of all events for each individual during the 48 hours of observation; 2) the same evaluation described for each period of 24hour recording, i.e., the first and the second periods according to each one of the recorded tapes; 3 ) the total number of events and the total period of time of ischemia in minutes for each period of 4 hours, in the 24 hours of each one of the two days of recording, respectively from 11:00 pm to 3:00 am, from 3:00 am to 7:00 am, from 7:00 am to 11:00 am, from 11:00 am to $3: 00 \mathrm{pm}$, from 3:00 pm to 7:00 pm, and from 7:00 pm to 11:00 pm.

Statistical analysis - the analysis of data was conducted by the Statistics Department of the Heart Institute. For measures that did not have a normal distribution, the variables were presented in a descriptive manner in graphs and tables containing the medians, means, standard deviations and minimum and maximum values, and the groups were evaluated by the Friedman's test ${ }^{14}$. This procedure was performed to evaluate the variation in the number of ischemic episodes and the duration of ischemia in the 4-hour intervals. Non Continuous variables were analyzed by the nonparametric Wilcoxon's rank sum test ${ }^{14}$, which was performed to compare the number of episodes and the duration of ischemia between the first and the second periods of the long-term electrocardiographic recording, i.e., the first tape recording compared with the second one.

The calculations were processed by the SAS (statistical analysis system) and a p value $<0.05$ was considered statistically significant.

\section{Results}

We observed 1755.8 hours of monitoring in the 39 patients, a mean of 45.02 (23.13 to 48 ) hours per case. We detected ischemic episodes in the long-term electrocardiograms of $18(46.2 \%)$ patients. In these 18 patients, the recordings allowed us to observe 820.88 hours of continuous electrocardiographic monitoring, corresponding to a mean of 45.6 \pm 5.76 ( 23.13 to 48 ) hours per patient (table I). In five patients, we could not obtain electrocardiogram tracines that allowed ST-segment analysis.

We detected 173 ischemic episodes in this observa- 
tion, allowing us to analyze a total of 1304 minutes of ischemia only 4 (2.2\%) of them being symptomatic. This corresponds to a mean of $9.61 \pm 10.66$ ( 1 to 43 ) ischemic episodes and $72.44 \pm 80.65$ (5.7 to 184.2$)$ minutes of ischemia per patient. Evaluating the occurrence of ischemic events, considering the whole period of recording and the predetermined intervals, we observed that there was a higher incidence of ischemic events from 11:00 am to 3:00 pm, either in the number, 38 episodes (fig. 1), or in the duration of ischemia, 315.4 minutes (fig. 2). However, no significant difference occurred among the values of these intervals, $p=0.47$ for the number of episodes and $\mathrm{p}=0.43$ for the period of time of ischemia. Similarly, a higher number of episodes and a longer period of time of ischemia occurred in the first period of recording, compared with the second (table I), without statistical significance, with $\mathrm{p}=0.42$ for the number of episodes and $p=0.35$ for the duration of ischemia. Figures 1 and 2 more effectively show the distribution of these events among the different intervals.

\section{Discussion}

Our results apparently show a relatively low incidence of myocardial ischemia in patients with unstable angina, considering that ischemia was present in less than $50 \%$ of the patients. Otherwise, our results confirm the high incidence of asymptomatic ischemic events $(97.8 \%)$ in a population of patients with unstable angina. Although our study population did not comprise a very large number of

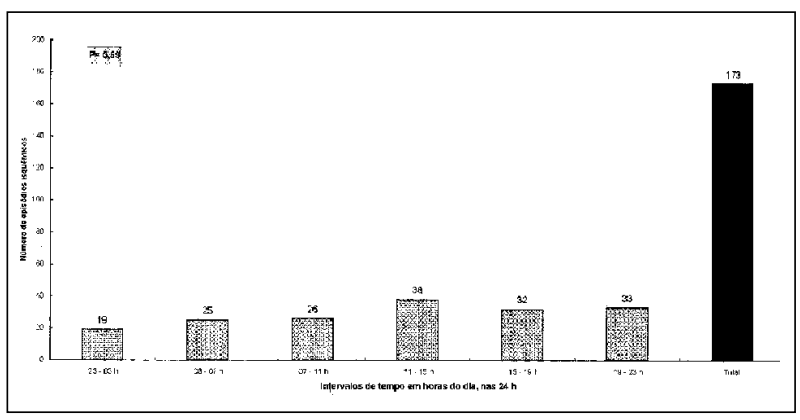

Fig. 1 - Number of ischemic episodes per time interval over 24 hours of a day.

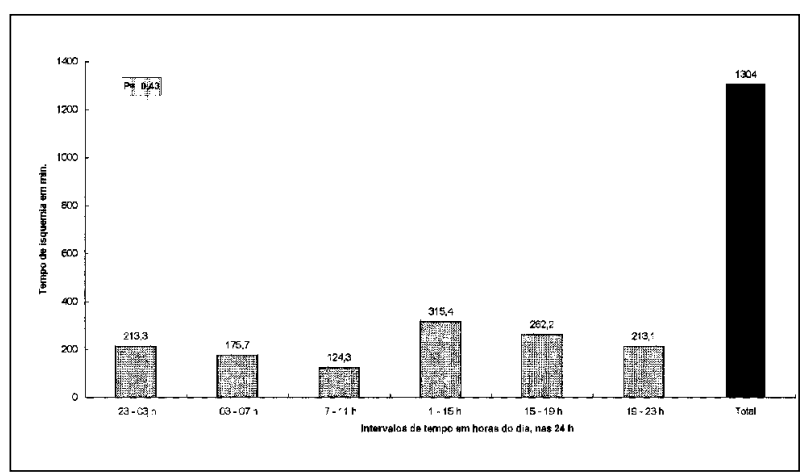

Fig. 2 - Duration of ischemia per time interval over 24 hours of a day.

patients, the use of long-term electrocardiography over 48 hours certainly diminished the importance of evaluating only 39 patients.

\begin{tabular}{|c|c|c|c|c|c|c|c|c|c|c|c|c|c|c|c|c|c|c|c|c|}
\hline \multirow{3}{*}{ Case } & \multicolumn{10}{|c|}{ Number of ischemic episodes } & \multicolumn{10}{|c|}{ Duration of ischemia in minutes } \\
\hline & \multicolumn{7}{|c|}{ 4-hour intervals } & \multicolumn{3}{|c|}{ 24-hour periods } & \multicolumn{7}{|c|}{ 4-hour intervals } & \multicolumn{3}{|c|}{ 24-hour periods } \\
\hline & $11 \mathrm{p}-3 \mathrm{a}$ & $3 a-7 a$ & $7 a-11 a$ & $11 a-3 p$ & $3 p-7 p$ & $7 p-11 p$ & Total & $1^{\text {st }}$ & $2^{\text {nd }}$ & Total & $11 \mathrm{p}-3 \mathrm{a}$ & $3 a-7 a$ & $7 a-11 a$ & $11 a-3 p$ & $3 p-7 p$ & $7 p-11 p$ & Total & $1^{\text {st }}$ & $2^{\text {nd }}$ & Total \\
\hline 1 & 0 & 0 & 1 & 0 & 2 & 0 & 3 & 2 & 1 & 3 & 0 & 0 & 9.7 & 0 & 41.9 & 0 & 51.6 & 41.9 & 9.7 & 51.6 \\
\hline 2 & 0 & 1 & 0 & 2 & 0 & 3 & 6 & 4 & 2 & 6 & 0 & 35.7 & 0 & 44.4 & 0 & 58.9 & 139 & 100.3 & 38.7 & 139 \\
\hline 6 & 1 & 5 & 0 & 0 & 3 & 2 & 11 & 9 & 2 & 11 & 13.2 & 13.7 & 0 & 0 & 25.7 & 9.2 & 61.8 & 52.1 & 9.7 & 61.8 \\
\hline 7 & 1 & 6 & 3 & 6 & 4 & 3 & 23 & 7 & 16 & 23 & 2.5 & 36.8 & 8 & 29.1 & 15.9 & 9.2 & 101.5 & 19.1 & 82.4 & 101.5 \\
\hline 10 & 1 & 0 & 1 & 7 & 3 & 2 & 14 & 13 & 1 & 14 & 1.7 & 0 & 4 & 78.4 & 33.2 & 24.4 & 141.7 & 127.5 & 14.2 & 141.7 \\
\hline 11 & 0 & 0 & 1 & 0 & 1 & 0 & 2 & 2 & 0 & 2 & 0 & 0 & 1 & 0 & 21.2 & 0 & 22.2 & 0 & 22.2 & 22.2 \\
\hline 12 & 1 & 5 & 4 & 4 & 1 & 3 & 18 & 6 & 12 & 18 & 10.5 & 19.1 & 20.4 & 41.7 & 8.5 & 9.8 & 110 & 61.4 & 48.6 & 110 \\
\hline 15 & 0 & 0 & 0 & 0 & 3 & 0 & 3 & 3 & 0 & 3 & 0 & 0 & 0 & 0 & 61.2 & 0 & 61.2 & 61.2 & 0 & 61.2 \\
\hline 18 & 0 & 0 & 2 & 0 & 5 & 1 & 8 & 8 & 0 & 8 & 0 & 0 & 8.5 & 0 & 9.6 & 2.2 & 20.3 & 20.3 & 0 & 20.3 \\
\hline 19 & 0 & 0 & 0 & 2 & 0 & 0 & 2 & 0 & 2 & 2 & 0 & 0 & 0 & 6 & 0 & 0 & 6 & 0 & 6 & 6 \\
\hline 20 & 0 & 3 & 4 & 2 & 3 & 3 & 15 & 14 & 1 & 15 & 0 & 4.7 & 15.2 & 7.5 & 10.4 & 6.2 & 44 & 42 & 2 & 44 \\
\hline 23 & 2 & 0 & 0 & 0 & 0 & 0 & 2 & 2 & 0 & 2 & 5.7 & 0 & 0 & 0 & 0 & 0 & 5.7 & 5.7 & 0 & 5.7 \\
\hline 29 & 0 & 0 & 0 & 0 & 0 & 1 & 1 & 0 & 1 & 1 & 0 & 0 & 0 & 0 & 0 & 17.7 & 17.7 & 0 & 17.7 & 17.7 \\
\hline 30 & 3 & 0 & 0 & 0 & 0 & 0 & 3 & 0 & 3 & 3 & 13.2 & 0 & 0 & 0 & 0 & 0 & 13.2 & 0 & 13.2 & 13.2 \\
\hline 33 & 6 & 0 & 6 & 11 & 6 & 14 & 43 & 20 & 23 & 43 & 81.6 & 0 & 27.4 & 97.7 & 30.1 & 73 & 309.8 & 174 & 135.8 & 309.8 \\
\hline 37 & 0 & 0 & 1 & 0 & 1 & 0 & 2 & 0 & 2 & 2 & 0 & 0 & 3.2 & 0 & 4.5 & 0 & 7.7 & 0 & 7.7 & 7.7 \\
\hline 38 & 0 & 0 & 0 & 3 & 0 & 0 & 3 & 2 & 1 & 3 & 0 & 0 & 0 & 6.4 & 0 & 0 & 6.4 & 3.7 & 2.7 & 6.4 \\
\hline 39 & 4 & 5 & 3 & 1 & 0 & 1 & 14 & 8 & 6 & 14 & 84.9 & 65.7 & 26.9 & 4.2 & 0 & 2.5 & 184.2 & 26.6 & 157.6 & 184.2 \\
\hline Total & 19 & 25 & 26 & 38 & 32 & 33 & 173 & 100 & 73 & 173 & 213.3 & 175.7 & 124.3 & 315.4 & 262.2 & 213.1 & 1304 & 735.8 & 568.2 & 1304.0 \\
\hline Mean & 1.06 & 1.39 & 1.44 & 2.11 & 1.78 & 1.83 & 9.61 & 5.56 & 4.06 & 9.61 & 11.85 & 9.76 & 6.91 & 17.52 & 14.57 & 11.84 & 72.44 & 40.88 & 31.57 & 72.44 \\
\hline$\pm \mathrm{SD}^{(1)}$ & 1.70 & 2.25 & 1.82 & 3.10 & 1.93 & 3.28 & 10.66 & 5.66 & 6.41 & 10.66 & 26.39 & 18.48 & 9.46 & 29.58 & 17.73 & 21.00 & 80.79 & 49.58 & 46.95 & 80.79 \\
\hline Median & 0.00 & 0.00 & 1.00 & 0.50 & 1.00 & 1.00 & 4.50 & 3.50 & 1.50 & 4.50 & 0.00 & 0.00 & 2.10 & 2.10 & 9.05 & 2.35 & 47.80 & 23.45 & 11.45 & 47.80 \\
\hline
\end{tabular}

Note: For comparisons among the intervals: $\mathrm{p}=0.59$ for episodes and $\mathrm{p}=0,43$ for duration of ischemia. For comparisons between periods: $\mathrm{p}=0.42$ for episodes and $\mathrm{p}=0.35$ for duration of ischemia. (1) standard deviation. 
Regarding the findings of the long-term electrocardiography as a function of the distribution of ischemic events throughout the 24 hours of a day, we should first point out that the option of dividing the day into 4-hour intervals and for defining them from 11:00 pm to 3:00 am, from 3:00 am to 7:00 am and so on, was based on the previous experience of other authors ${ }^{8,9,12,13,15-17}$, who reported, as a rule, a higher frequency of ischemic events from dawn until the first hours in the morning, the so-called circadian variation of ischemia, with or without symptoms. This evidence was based on studies that evaluated the incidence of acute myocardial infarction throughout the 24 hours of a day ${ }^{18,19}$, which showed a higher incidence between 3:00 am and 8:00 am. We realized, therefore, that, using these intervals of time, we would be able to detect a possible higher incidence of myocardial ischemia during the dawn (3:00 am to 7:00 am) period and in the morning (7:00 am to 11:00 am) period. However, our data showed a higher incidence of ischemic episodes and a longer duration of ischemia from 11:00 am to $3: 00 \mathrm{pm}$, based on the sum of episodes of the two recorded tapes, i.e., during the 48 hours of the examination (table I). Although the comparative analysis did not demonstrate any significant difference in the incidence of ischemic episodes amon the intervals, the graphic observation of the events (figures 1 and 2) shows a trend toward higher incidence of events in the period between 11:00 am and 11:00 pm, compared with the period between 11:00 pm and 11:00 am. This was a surprising finding because it was totally opposite to the previous reports in the literature cited above. We can conjecture that our observation was made with a relatively small number of patients whereas others studied up to 193 patients ${ }^{12}$ to conclude about this circadian variation in angina and myocardial ischemia. We can also question whether this distribution had some meaning in our patients, once the statistical analysis failed to show significant differences among the intervals of time.

To clarify this doubt, some points of concern need to be addressed. In the report of Figueiras et al ${ }^{10}$, in 198616 patients with resting angina and severe coronary artery disease were evaluated to determine the angina threshold at different times of the day. In the evaluation of this threshold, the heart rate needed to cause an ischemic episode was used, during at least six observations and for up to 4 days. The elevation in the heart rate was obtained through a cardiac pacemaker connected to the right atrium by puncture of the right subclavian vein. The authors decided to perform the stimuli in the following periods: between 10:00 pm and 1:00 am, 4:00 pm and 7:00 pm and midnight and 3:00 am. Analyzing the results, the authors concluded that "patients with resting angina and severe coronary artery disease frequently exhibit a fall in the ischemic threshold during the night". The night period referred to by the authors corresponds to the period between 10:00 pm and 1:00 am. In 1995, Figueiras and Lidón ${ }^{13}$ used the same technique to evaluate the ischemic threshold in patients with unstable angina and severe coronary artery disease. However, the authors chose other periods of time for their observations (from 7:00 am to
8:00 am and from midnight to 1:00 am) and concluded that the reduction in the ischemic threshold in the beginning of the morning, in the absence of elevation in the baseline heart rate and blood pressure, suggests that a reduction in the coronary vasodilation capacity or an elevation in the coronary tonus may favor a higher incidence of ischemic events during this interval. The conclusion of this study makes it evident that different results can be obtained depending on the time the observation is made. The doubt then arises when one wants to know the exact time of day when a lower ischemic threshold exist, and consequently an increase in the incidence of ischemic events. Analyzing another study ${ }^{8}$ in which the authors evaluated 32 patients with stable angina continuously monitored for at least 24 hours, we verified, through the figures in the manuscript, that the time with the highest incidence of ischemia (related to physical effort) was from 6:00 am to noon. However, analyzing the same figures, another period with an increase in ischemic episodes can be noted, specifically from noon to 3:00 pm. Finally, in another study, Figueiras and Lidón observed ischemic episodes through the occurrence of anginal symptoms in two periods of the day, between 7:00 am and 11:00 am and 7:00 pm and 11:00 pm, in 193 patients with unstable angina, a very expressive number of patients. The analysis of subgroups showed a higher incidence of angina between 11:00 pm and 2:00 am in patients with severe, multivessel coronary artery disease.

In summary, although considerable evidence exist suggesting that ischemic episodes occur more often in the dawn and morning hours, particularly in the presence of a clinical condition of unstable angina, we should consider the possibility that populational variations can affect the occurrence of such episodes. In our population, Solimene et $\mathrm{al}^{20} \mathrm{did}$ not find variations in the occurrence of ischemia during the 24 hours of a day, analyzing patients some months after an acute myocardial infarction. In addition, two other two researchers ${ }^{21,22}$ did not observe circadian rhythm and morning ischemia after an acute myocardial infarction either. On the contrary, they noted an even a higher incidence of ischemia from 6:00 pm to midnight. Finally, it is worth noting the experience of Beamer et al ${ }^{11}$. These authors studied 3990 individuals with a history of chest pain admitted to the Emergency Department of seven different hospitals. Their findings confirmed a higher number of diagnoses of acute myocardial infarction and unstable angina between 6:00 am and noon, but chest pain that was caused by nonischemic cardiac causes and by noncardiac causes was also more likely to begin during the same time period. It seems, therefore, that this circadian rhythm is related to factors that can trigger chest pain, not restricted to ischemic myocardial pain.

Therefore, our patients with unstable angina showed a high incidence $(97.8 \%)$ of silent ischemic episodes, evaluated by long-term electrocardiography over 48 hours. We did not find any evidence of a circadian variation in myocardial ischemia in unstable angina, unlike the majority of previous studies, probably because of populational va- 
riations. In addition, we noted a trend suggesting a higher distribution of episodes and a longer duration of ischemia in the afternoon period, especially between 11:00 am and 3:00 $\mathrm{pm}$, and not in the morning period.

\section{References}

1. Stern S, Tzivoni D. Early detection of silent ischaemia heart disease by 24 hours electrocardiographic monitoring of active subjects. Br Heart J 1974; 36; 481-5.

2. Allen RD, Gettes LS, Phalan C, Avington D. Painless ST-depression in patients with angina pectoris. Chest 1976; 69: 467-73.

3. Epstein SE, Quyyumi AA, Bonow RO. Myocardial ischaemia: silent or symptomatic. NEngl J Med 1988; 318: 1038-43.

4. Solimene MC. Isquemia silenciosa. Rev Soc Cardiol ESP 1993; 3: 54-9.

5. Parmley WW. Prevalence and clinical significance of silent myocardial ischaemia. Circulation 1989; 80: 68-73.

6. Johnson SM, Mauritson DR, Winniford MD, et al. Continuous electrocardiographic monitoring in patients with unstable angina pectoris: identification of high-risk subgroup with severe coronary disease, variant angina and/or impaired early prognosis. Am Heart J 1982; 103: 4-12.

7. Gottleib SO, Weisfeldt ML, Ouyang P, Mellits ED, Gerstenblith G. Silent ischaemia as a marker for unfavorable outcomes in patients with unstable angina. $\mathrm{N}$ Engl J Med 1986; 314: 1214-9.

8. Rocco MB, Barry J, Campbell S, et al. Circadian variation on transient myocardial ischemia in patients with coronary artery disease. Circulation 1987; 75: 395 400 .

9. Nademanee K, Intarachot V, Josephson MA, Singh BN. Circadian variation in occurence of transient overt and silent myocardial ischemia in chronic stable angina and comparasion with Prinzmetal angina in men. Am J Cardiol 1987; 60: 494-8.

10. Figueras J, Cinca J, Balda F, Moya A, Rius J. Resting angina with fixed coronary artery stenosis: nocturnal decline in ischemic threshold. Circulation 1986; 74: 1248-54.

11. Beamer AD, Lee TH, Cook EF, et al. Diagnostic implications for myocardial ischemia of the circadian variation of the onset of chest pain. Am J Cardiol 1987; 60: 998-1002.

12. Figueras J, Lidón RM. Circadian rythm of angina in patients with unstable angi- na: relationship with extent of coronary disease, coronary reserve and ECG changes during pain. Eur Heart J 1994; 15: 753-60.

13. Figueras J, Lidón RM. Early morning reduction in ischemic threshold in patients with unstable angina and significant coronary disease. Circulation 195; 92: 1737-42.

14. Hollander M, Wolfe DA. Nonparametric Statistical Methods. New York: John Wiley \& Sons Inc., 1973: 68-138.

15. Yasue H, Omote S, Takizama A, Nagao M, Miwa K, Tanaka S. Circadian variation of exercise capacity in patients with Prinzmetal variant angina: role of exerciseinduced coronary arterial spasm. Circulation 1979; 59: 938-48.

16. Haussmann D, Nikutta P, Trappe H, Daniel WG, Wendzlaff P, Lichtlen PR. Circadian distribution of characteristics of ischemic episodes in patients with stable coronary artery disease. Am J Cardiol 1990; 66: 668-72.

17. Carpeggiani C, Michelassi M, Biagini A, et al. Findings from long-term electrocardiographic monitoring of patients with variant angina. Am J Cardiol 1987; 60: 36-9.

18. Thompson DR, Blandford RL, Sutton TW, Merchant PR. Time of onset of chest pain in acute myocardial infarction. Int J Cardiol 1985; 7: 139-46.

19. Muller JE, Stone PH, Turi ZG, et al, and MILIS Study Group. Cicadian variation in the frequency of onset of acute myocardial infarction. NEngl J Med 1985;313: 1315-22.

20. Solimene MC, Ramires JAF, Gruppi CJ, Oliveira SFO, da Luz PL, Pilegi F. Variabilidade da freqüência cardíaca e isquemia silenciosa após infarto do miocárdio. Arq Bras Cardiol 1991; 57: 363-70.

21. Currie P, Saltissi S. Transient myocardial ischemia after acute myocardial infarction. Br Heart J 1991; 64: 299-303.

22. Mickley H, Pless P, Nielsen JR, Moller M. Changing circadian variation of transi-ent myocardial ischemia during the first year after a first myocardial infarction. Am J Cardiol 1992; 64: 1117-22. 